\title{
Possible traces of solar activity effect on the surface air temperature of mid-latitudes
}

\author{
Ali Kilcik ${ }^{1}$, Atila Özgüç ${ }^{2}$ and Jean-Pierre Rozelot ${ }^{3}$ \\ ${ }^{1}$ Department of Physics, Faculty of Science, Akdeniz University, 07058 Antalya, Turkey \\ email: kilcikali@gmail.com \\ ${ }^{2}$ Kandilli Observatory and E.R.I., Bogazici University, Cengelkoy, 34684 Istanbul, Turkey \\ email: ozguc@boun.edu.tr \\ ${ }^{3}$ OCA-Fizeau Dpt, CNRS UMR 6525, \& Université de Nice-Sophia-Antipolis, \\ Av. Copernic, 06130 Grasse, France \\ email: rozelot@obs-azur.fr
}

\begin{abstract}
In this study we investigate the effects of solar activity on the surface air temperature of mid-latitudes. This enables us to understand existence of solar activity effects on the temperature. We used surface air temperature and pressure data as climate parameters, and solar flare index data as solar activity indicator, for the 25 - 50 degree longitude and 30 - 70 degree latitude zone, including Turkey and European part of Russia. We considered the parameters temperature, pressure and flare index data for the period ranging from January 1975 to the end of December 2007, which covers almost three solar cycles, namely $21^{s t}, 22^{n d}$, and $23^{r d}$. We found some significant correlations between solar activity and surface air temperature for cycles 22 and 23 for some zones. We applied multitaper method to obtain the cyclic behavior of surface air temperature data sets. The most pronounced power peaks were found by this transform around 1.2 and 2.5 years which were reported earlier for some solar activity indicators. We concluded that signature of solar activity effect exists on surface air temperature of mid-latitudes where we studied.
\end{abstract}

Keywords. Sun: activity, flares, solar-terrestrial relations, methods: data analysis

\section{Introduction}

There is a great deal of interest in studying links between solar activity and changes in Earths climate. It has been known for some time that there is a statistical relation between solar activity as expressed, for instance, by the sunspot number or flare index, and the surface air temperature (see Fig. 1). Much scientific effort has been exercised in trying to understand and quantify how much influence the Sun exerts on Earths climate. Here, we highlight some of the recent scientific researches on such solar linkages with surface air temperature. For example, Valev (2006) found statistically significant correlations between global and hemispheric temperature anomalies and solar and geomagnetic indices from 1856 to 2000. Kilcik (2005) compared the solar irradiance model data to surface air temperature variations of two countries: USA and Japan and showed the influence of solar activity variations on the Earth's climate. Recently Kilcik et al. (2008) investigated the effects of solar activity on the surface air temperature of Turkey to understand existence of solar activity effects on the temperature. They found significant correlation coefficients between the two parameters. This put in evidence that the relationship between solar activity and surface air temperature is very complicated and varies with time and probably also with the considered geographic area. On a global scale, the correlation between solar activity indices and surface air temperature may be 

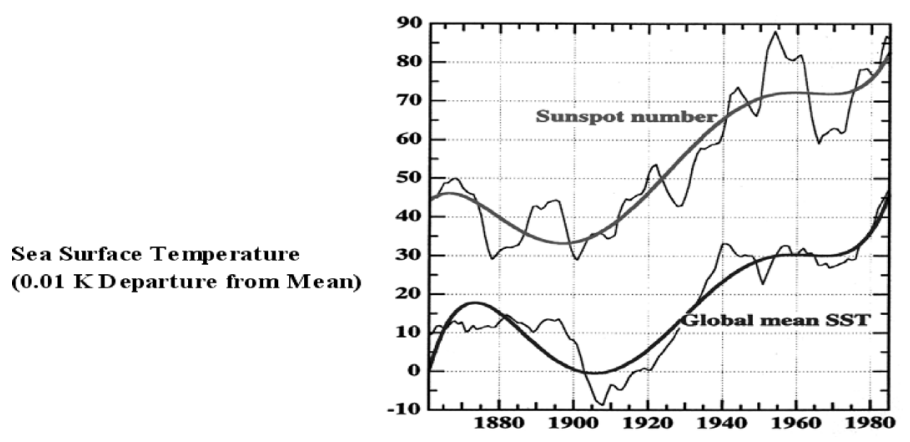

Figure 1. The correlation between the solar and climate records can be seen in this figure comparing polynomial fits to the sunspot record and the global mean sea-surface temperature SST. The Similarity is remarkable (after NOAA Space Environment Center).

positive, negative, or even zero. The correlation is always different for different areas observed (Zhang et al. 1989). For example the existence of a positive relation between the surface air temperature of the Northern hemisphere and the solar activity in the period 18811988 has been shown by Georgieva (1998). In this study high positive correlation was found between the geomagnetic activity and the surface air temperature in Middle and Southern Europe However, the same correlation over Canada area is negative (Bucha and Bucha Jr., 1998). In this paper, we attempt to find the effects of a specific solar activity index over a well-defined area. We specify the data used and describe the methods of analysis in Section 2. The results are given in Section 3, while discussion and conclusion remarks are reported in Section 4.

\section{Data and Methods}

Monthly Solar flare index data set is used as solar activity indicator. The flare index data, which we used, are taken from Kandilli Observatory website† web site. Flare index introduced by Kleczek (1952) is the quantity of Q = i t, where i represents the intensity scale of importance of a flare $\mathrm{Ha}$ and the duration (in minutes) of the flare. The determination of Q has been explained previously (Özgüç et al., 2003, 2004). The anomalies of the temperatures for four zones and these of the solar flare index are shown in Fig. 4.

To investigate the existence of any relation between yearly solar flare index and four zones temperature data, Pearson correlation analyses were applied to the entire yearly data and yearly data set and then we fragmented the temperature and the flare index data according to the solar cycles and again Pearson correlation is applied to these fragmented zonal yearly temperature and flare index data sets. The correlation coefficients for the entire data and for the four zones are presented in Table 2. In order to compare the spectral characteristics of the flare index to the spectral characteristics of surface air temperature we used multitaper method (MTM), which is developed by Thomson (1982) for reducing the variance of spectral estimates by using a set of tapers. In this study we used three sinusoidal tapers. This method has been previously successfully used for the analysis of climate time series of different time scale and resolution (Ghil and Vautard, 1991; Yiou et al., 1996; Anandan et al., 2004; Kilcik et al. 2008). Since this method is now standard, and can be found elsewhere, we do not describe it here. To analyze our data the frequency range from 0.00 to 0.078 for solar flare index data, and from 0.005 to

$\dagger$ http://www.koeri.boun.edu.tr/astronomy/astronomy.html 


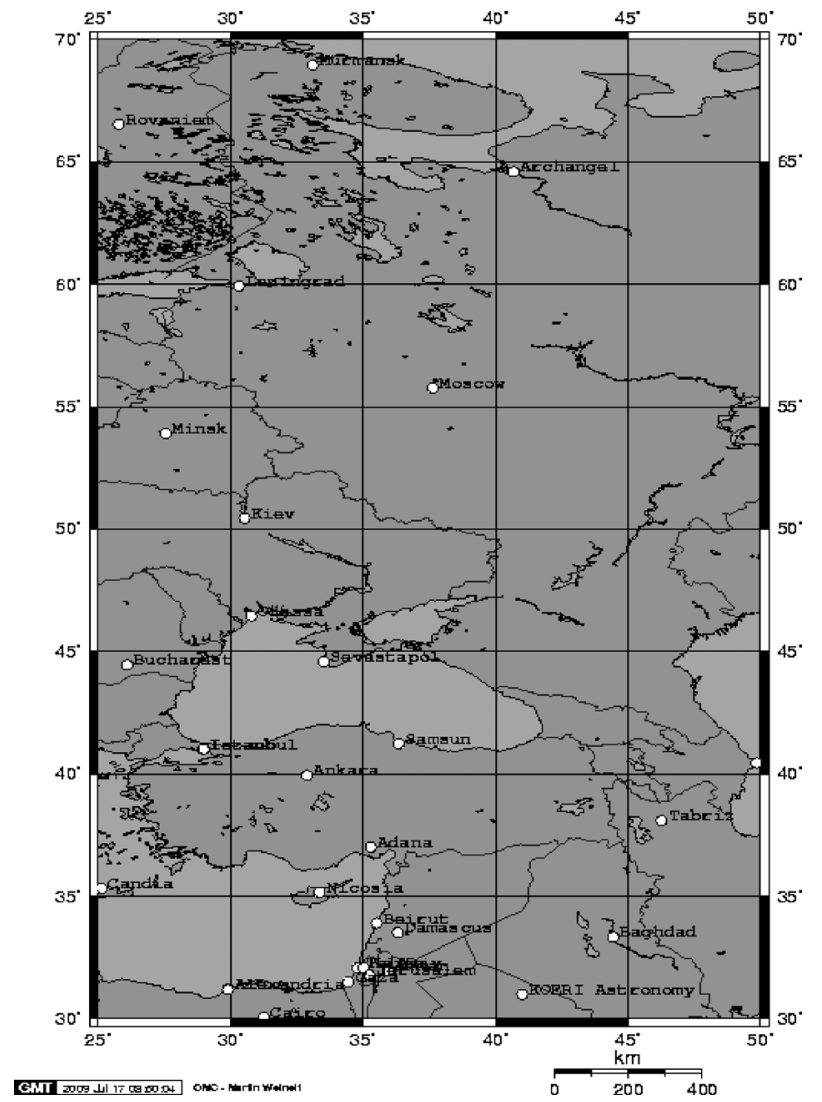

Figure 2. Map of the investigated geographic regions used in this study.

0.078 for all temperature data used in this study were selected. Our significance test is carried out with respect to red noise, since the temperature records, like most climatic and other geophysical time series, have larger power at lower frequencies. All harmonic signals have been obtained by using of $95 \%$ confidence level.

\begin{tabular}{ll}
\hline Latitudinal zones & Number of stations \\
\hline $36-42$ (Turkey) & 23 \\
$40-50$ & 5 \\
$50-60$ & 15 \\
$60-70$ & 4 \\
\hline
\end{tabular}

Table 1. Four latitudinal zones and the number of stations for each zone.

\section{Analyses and Results}

To investigate Sun climate relation for the geographic regions under consideration, we compare the general behavior of solar flare index and four zones temperature anomalies in the first step. These comparisons are given in Figure 4. As shown in this figure, a 


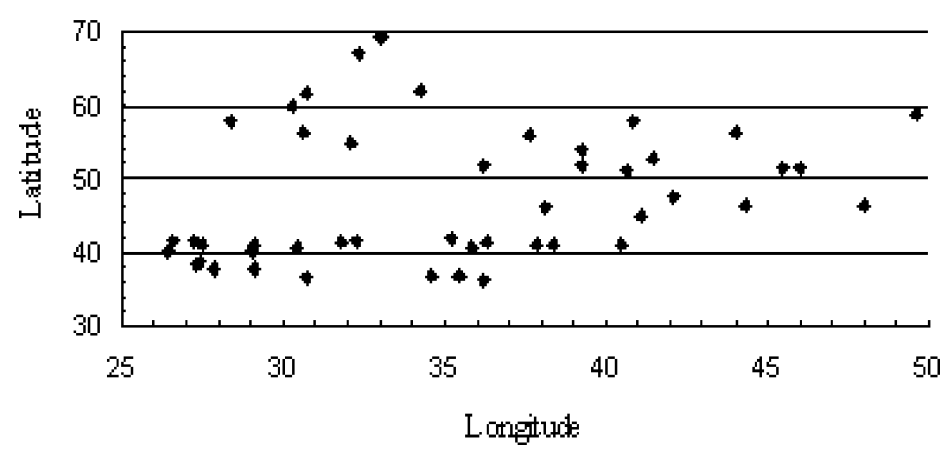

Figure 3. Distribution of the stations used in this study.

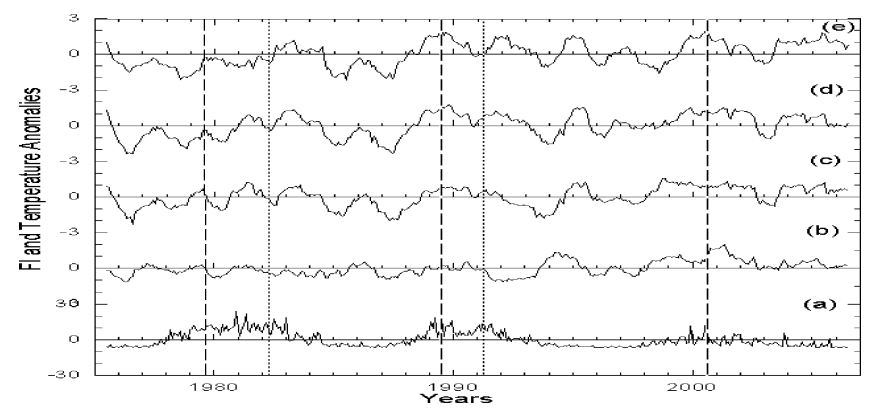

Figure 4. Comparison of the temperature anomalies of four zones with solar flare index anomalies. The temperature anomaly values are given in Celsius. Dashed vertical lines mark the solar activity cycle maxima, according to NOAA mean monthly sunspot numbers. Dotted vertical lines mark the beginning of the two volcanic activities, El Chichon and Pinatuba, respectively. In the figure (a) solar flare index, (b) 30-40 degree (Turkey), (c) 40-50 degree, (d) 50-60 degree, and (e) 60-70 degree zonal anomalies are shown respectively.

good agreement is found between solar flare and four regions temperature anomalies, during the period of cycles maxima especially for cycle 22 and 23. Correlation analyses between yearly solar flare and temperature data were applied to each cycle separately. In the results of our correlation analyses we could not find a statistically significant correlation between yearly mean surface air temperature of all regions and solar flare index data sets from 1976 to 2007. From the correlation analyses results, there is no significant correlation between the two data sets for cycle 21 (1976-1986). We obtained a significant correlations for 50-60 and 60-70 degree of latitude, but we could not obtained a significant correlations for 3040 and 40-50 degree latitude intervals for cycle 22 (19861996). For cycle 23 (1996-2006) we could not obtain any significant correlation for 60-70 degree latitude interval. All data correlation coefficients are given in Table 2 .

In order to compare the spectral characteristics of the flare index to the spectral characteristics of surface air temperature we used MTM harmonic test. Our spectral analyses results are given in Figure 5 and 6 for monthly solar flare index and four zonal temperature data respectively. Our periodogram analysis results show that all zonal temperature data sets have all about the same periodicity, showing some small differences. 


\begin{tabular}{ccccc}
\hline $30^{\circ}-40^{\circ}$ & $40^{\circ}-50^{\circ}$ & $50^{\circ}-60^{\circ}$ & $60^{\circ}-70^{\circ}$ & \\
\hline & & & & \\
-0.09284 & 0.12881 & 0.18595 & 0.04499 & Full data \\
0.12364 & 0.22086 & 0.12770 & -0.02867 & Cycle 21 \\
-0.17344 & 0.34993 & $\mathbf{0 . 6 0 8 9 9}$ & $\mathbf{0 . 6 4 2 8 5}$ & Cycle 22 \\
$\mathbf{0 . 7 6 7 3 2}$ & $\mathbf{0 . 5 2 0 1 9}$ & $\mathbf{0 . 6 3 7 0 0}$ & 0.06950 & Cycle 23
\end{tabular}

Table 2. Correlation coefficients of the entire and cyclic yearly mean data sets. Significant coefficients are given in bold face type.

Monthly Fare Index MTM Power Spectrum

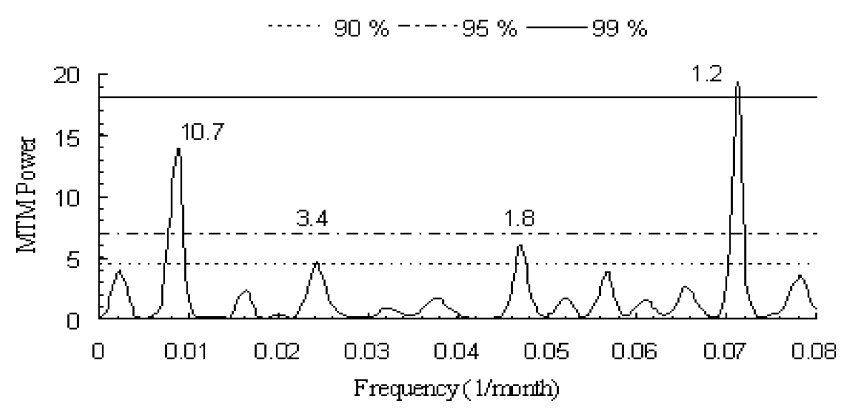

Figure 5. Multitaper method analysis of the 33 years long of the solar flare index from 1975 to 2007. Horizontal lines show the significant levels corresponding to red noise spectrum at 90, 95, and $99 \%$ confidence limits.

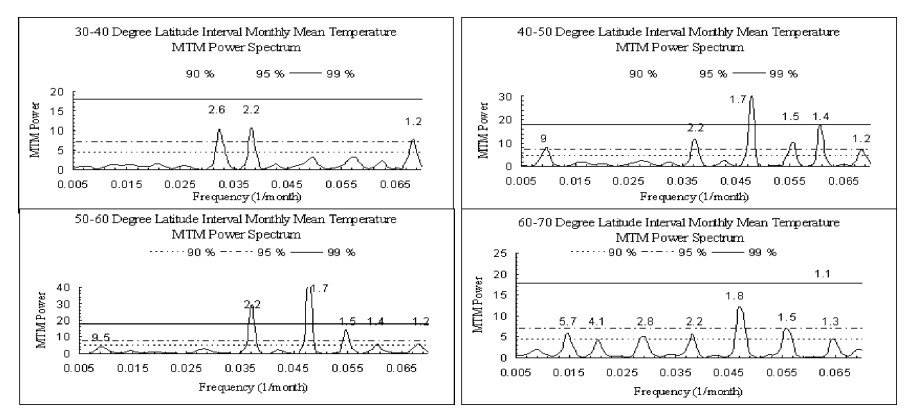

Figure 6. Multi taper method analysis of the 33 years long surface air temperature of the four zones from 1975 to 2007 . Horizontal lines show the significant levels corresponding to red noise spectrum at 90,95 , and $99 \%$ confidence limits.

\section{Conclusion and discussion}

The comparisons we have outlined imply that solar cycle variability may therefore play a significant role in surface air temperature in some mid latitudinal zones. As shown in Table 2 all the considered zones have not any significant correlation for the cycle 21, but for cycles 22 and 23 there are some meaningful correlations; $40-50$ and 50-60 degree latitude intervals temperature data show better positive correlations than other two intervals. In results of our correlation analyses we may assume the existence of low degree latitude dependencies of sunclimate relation. As shown in Figure 4, there is an increasing tendency in all zones temperature anomalies for the whole investigated time period point of view. These increases may arise from the enormous increasing of the amount of atmospheric greenhouse gases (GHGs) (CO2, CH4, NO2, etc.) concentrations. 


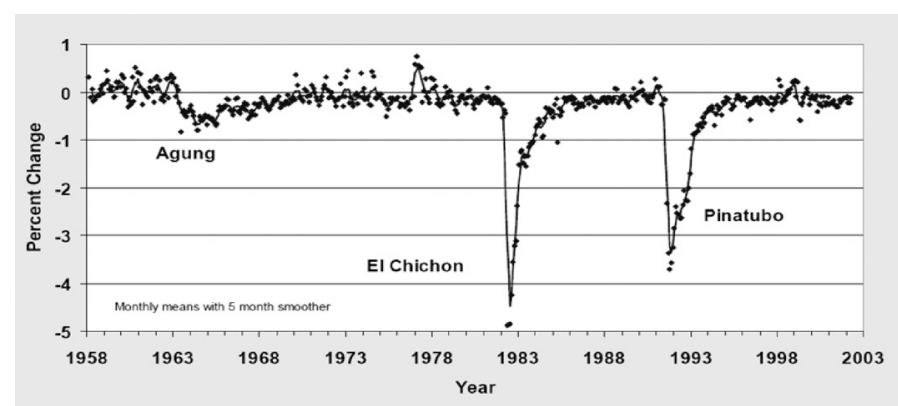

Figure 7. Net solar radiation measured at Mauna Loa Observatory, relative to 1958, showing the effects of major volcanic eruptions. Annual variations are due to the transport of Asian dust and air pollution to Hawaii. Figure is taken from Earth System Research Laboratory web page (http:// www.esrl.noaa.gov/gmd/about/climate.html).

FI and temperature anomalies show an opposite behavior from 1982 to 1985 (see figure 4). It is interesting to note that at the same time period there was strong volcanic activity (March 28, 1982, El Chichon (Mexico); 17.4N, 93.2W) and as seen in Figure 7, the net solar radiation decreased remarkably as measured at Mauna Loa observatory in Hawaii $(19.54 \mathrm{~N}, 155.58 \mathrm{~W})$. A close look at Figure 4 reveals that the temperature anomalies show really deep valley from 1991 to 1994 and again, this time period is in accordance with another strong volcanic activity (April 2, 1991, Pinatubo (Mexico); 15.13N, 120.35E). Also the net solar radiation decrease is measured at Mauna Loa Observatory and can be seen clearly in Figure 7 . As can be seen from Figure 4 it is interesting to note that the volcanic aerosol effects depend on latitude; in the higher latitudes the effect is seen later than the lower ones. These results indicate that GHGs and stratospheric volcanic aerosols have a remarkable effect on temperature behaviors.

There are two meaningful groups of periods for the surface air temperature, which are at 1.3-1.8 and 2.4-2.6 years (see fig.5 and fig.6) The first group found in our study was reported by different authors, such as Kirivova and Solanki (2002) when they analyzed the sunspot areas and the sunspot number data, respectively for the 1880-2000 and the 1750-2000 time periods. Such a period of 1.3-year can be a sub-harmonic of other periodic effects including the well known Schwabe cycle (10.5-year). This issue may be related to the quasi-biennial oscillations occurring in the stratospheric winds. Obridko and Shelting (2007) recently reported that oscillations of 1.3 -yr period are closely associated with quasi-biennial oscillations of large-scale solar magnetic field. We may assume this as another possibility. Kilcik et al (2008) also reported same periodicities from surface air temperature of Turkey and flare index for the 1975-2006 time period. In addition our spectral analysis of 60-70 degree zone shows 5.7 years periodicity. This period may be related to geomagnetic activity (5.25-year) and of course indirectly to solar activity. However using the geomagnetic activity index aa, Kane (1997) found a QBO and a 5.4years periodicity during the period of time 1868-1994. This periodicity was also reported in the biological and biophysical studies (Faraone et al., 2001; Kovac and Mikulecky, 2005; Dimitrova, 2008).

From the correlative and spectral analyses of 33 years data of the flare index and the surface air temperature of some mid latitude zones, conclusions of the present study are:

- Signatures of solar activity effect exist on surface air temperature of some mid-latitude regions according to our statistical analysis and over the considered period of time.

- Change of stratospheric volcanic aerosol concentration has a remarkable effect on surface air temperature. 
- Change of atmospheric greenhouse gases concentrations have remarkable effect on temperature.

\section{Acknowledgements}

One of the authors (A. Ö.) expresses his thanks to the organizers of the IAU symposium 264 for permitting to attend the IAU General Assembly. This work was supported by the Scientific and Technical Council of Turkey under project number $107 \mathrm{~T} 878$.

\section{References}

Bucha, V., Bucha Jr., \& V., 1998, JASTP 60, 145

Dimitrova, S., 2008, JASTP 70, 420

Faraone, P., Cornelissen, G., Katinas, G. S., Halbergi, G. F., \& Siegelova, J., 2001, Scripta Medica 74, 107

Georgieva, K., 1998, Bulg. Geophys.J. 3, 114

Ghil, M. \& Vautard, R., 1991, Nature 350, 324

Kane, R. P., 1997, Ann. Geophys. 15, 1581

Kilcik, A., 2005, JASTP 67, 1573

Kilcik, A., Özgüç, A., Rozelot, J. P., \& Yesilyurt, 2008, JASTP 70, 1669

Kovac, M., Mikulecky, M. S., 2005, Bratislavske Lekarske Listy 106, 423

Krivova, N. A. \& Solanki, S. K., 2002, Astron. Astrophys. 394, 701

Obridko, V. N. \& Shelting, B. D., 2007, Adv.Space Res. 40, 1006

Özgüç, A., Atac, T., \& Rybak, J. 2003, Solar Phys., 214, 375

Özgüç, A., Atac, T., \& Rybak, J. 2004,Solar Phys., 223, 287

Rigozo, N. R., Nordemann, D. J. R., Souza Echer, M. P., Echer, E., da Silva, H. E., Prestes, A., \& Guarnieri, F. L., 2007, JASTP 69, 1049

Rozelot, J. P., 1990 In "New approaches in Geomagnetism and the Earth's Rotation", ed. S. Flodmark, World Scientific, London, pp. 245-253

Thomson, D. J., 1982, IEEE Proceedings 70, 1055

Valev, D., 2006, Phys. Chem. Earth 31109

Yiou, P., Baert, E., \& Loutre, M. F., 1996,Survey in Geophysics 17, 619

Zhang Y. D. \& Li W. B., 1989, Sunspot Numbers, Beijing: Overseas Chinese Press, 139 